Proyecciones Journal of Mathematics

Vol. 32, No 3, pp. 267-280, September 2013.

Universidad Católica del Norte

Antofagasta - Chile

\title{
On some seminormed sequence spaces defined by Orlicz function
}

\author{
M. Aiyub \\ University of Bahrain, India \\ Received: September 2012. Accepted: May 2013
}

\begin{abstract}
The sequence space $B V_{\sigma}$ was introduced and studied by Mursaleen [9]. In this paper we extend $B V_{\sigma}$ to $B V_{\sigma}(M, p, q, r)$ on a seminormed complex linear space by using orlicz function. We give various properties and some inclusion relations on this space.
\end{abstract}




\section{Introduction}

Let $\ell_{\infty}, c$ and $c_{0}$ be respectively the Banach spaces of bounded, convergent and null sequences $x=\left(x_{k}\right)$ with $\left(x_{k}\right) \in \mathbf{R}$ or $\mathbf{C}$ the usual norm $\|x\|=\sup _{k}\left|x_{k}\right|$, where $k \in \mathbf{N}=1,2,3 \ldots$, the positive integers. Let $\sigma$ be an injection of the set of positive integers $\mathbf{N}$ into itself having no finite orbit and $T$ be the operator defined on $\ell_{\infty}$ by $T\left(\left(x_{n}\right)_{n=1}^{\infty}\right)=\left(x_{\sigma(n)}\right)_{n=1}^{\infty}$.

A positive linear functional $\phi$ with $\|\phi\|=1$ is called a $\sigma$-mean or an invariant mean if $\phi(x)=\phi(T x)$ for all $x \in \ell_{\infty}$

A sequence $x$ is said to be $\sigma$-convergent, denoted by $x \in V_{\sigma}$, if $\phi(x)$ takes the same value, called $\sigma-\lim x$, for all $\sigma$-means $\phi$.(Schaefer [14])

$$
V_{\sigma}=\left\{x=\left(x_{n}\right): \sum_{m=1}^{\infty} t_{m, n}(x)=L \text { uniformly in } n, L=\sigma-\lim x\right\} .
$$

Where $m \geq 0, n \geq 0$

$$
t_{m, n}(x)=\frac{x_{n}+x_{\sigma(n)}+\ldots .+x_{\sigma^{m}(n)}}{m+1}, \quad \text { and } t_{-1, n}=0 .
$$

Where $\sigma^{m}(n)$ denotes the $m$ - iterative of $\sigma$ at $n$. In particular, if $\sigma$ is the translation a $\sigma$-mean is often called a Banach limit and $V_{\sigma}$ reduces to $f$, the set of almost convergent sequence [5]. Subsequently invariant means have been studied by Ahmad and Mursaleen [1], Mursaleen [8], Rami [12] and many others.

The concept of paranormed is closely related to linear metric spaces. It is generalization of that of absolute value. Let $X$ be a linear space. A function $g: X \rightarrow R$ is called paranorme, if

$(P 1) g(x) \geq 0$, for all $x \in X$,

(P2) $g(-x)=g(x)$, for all $x \in X$, 
(P3) $g(x+y) \leq g(x)+g(y)$, for all $x, y \in X$,

(P4) if $\lambda_{n}$ is a sequence of scalar with $\lambda_{n} \rightarrow \lambda(n \rightarrow \infty)$ and $\left(x_{n}\right)$ is a sequence of vector with $g\left(x_{n}-x\right) \rightarrow 0(n \rightarrow \infty)$ then $g\left(\lambda_{n} x_{n}-\lambda x\right) \rightarrow$ $0(n \rightarrow \infty)$.

A paranormed $g$ for which $g(x)=0$ implies $x=0$ is called a total paranorm on $X$ and pair $(X, g)$ is called a totally paranormed space. It is well known that the metric of any linear metric space is given by total paranorm (cf [15, Theorem 10.4.2, p-183]).

A map $M: R \rightarrow[0,+\infty]$ is called to be an orlicz function if $M$ is even, convex left continuous on $R_{+}$, continuous at zero, convex $M(0)=0$ and $M(u) \rightarrow \infty$ as $u \rightarrow \infty$.

If $M$ takes the value zero only at zero we write $M>0$ and if $M$ takes only finite value we will write $M<\infty$. [2,3,6,7,10,13]

W. Orlicz [11] used the idea of orlicz function to construct the space $\left(L^{M}\right)$ Lindenstrauss and Tzafriri [4] use the idea of Orlicz function and defined the sequence space $\ell_{M}$ such as

$$
\ell_{M}=\left\{x=\left(x_{i}\right): \sum_{i=1}^{\infty} M\left(\frac{\left|x_{i}\right|}{\rho}\right)<\infty, \text { for some } \rho>0\right\} .
$$

The space $\ell_{M}$ with the norm $\|x\|=\inf \left\{\rho>0: \sum_{k=1}^{\infty} M\left(\frac{\left|x_{k}\right|}{\rho}\right) \leq 1\right\}$ becomes a Banach space which is called an Orlicz sequence space. The space $\ell_{M}$ is closely related to the sequence space $\ell_{p}$, which is an Orlicz sequence spaces with $M(x)=x^{p}$ for $1 \leq p \leq \infty$.

The $\Delta_{2}$-condition is equivalent to $M(L x) \leq K L M(x)$, for all $x \geq 0$ and for $L>1$.

An Orlicz function $M$ can be represented in the following integral from

$$
M(x)=\int_{0}^{x} \eta(t) d t,
$$

where $\eta$ is known as the kernal of $M$ is right differentiable for $t \geq 0, \eta(0)=$ 
$0, \eta(t)>0, \eta$ is non- decreasing and $\eta(t) \rightarrow \infty$ as $t \rightarrow \infty$. Note that an Orlicz function satisfies the inequality

$$
M(\lambda x) \leq \lambda M(x) \text { for all } \lambda \text { with } 0<\lambda<1 .
$$

Let $E$ be a sequence space. Then $E$ is called

(i) A sequence space $E$ is said to be symmetric if $\left(x_{n}\right) \in E$ implies $\left(x_{\pi(n)}\right) \in E$, where $\pi(n)$ is a permutation of the elements of $\mathbf{N}$,

(ii) Solid (or normal), if $\left(\alpha_{k} x_{k}\right) \in E$, whenever $\left(x_{k}\right) \in E$ for all sequences of scalar $\left(\alpha_{k}\right)$ with $\left|\alpha_{k}\right| \leq 1$ for all $k \in \mathbf{N}$.

Lemma 1.1. A sequence space $E$ is solid implies $E$ is monotone.

Mursaleen [9] defined the sequence space.

$$
B V_{\sigma}=\left\{x \in \ell_{\infty}: \sum_{m}\left|\phi_{m, n}(x)\right|<\infty \text { uniformly in } n\right\}
$$

Where

$$
\phi_{m, n}(x)=t_{m, n}(x)-t_{m-1, n}(x)
$$

assuming

$$
t_{m, n}(x)=0, \text { for } m=-1 \text {. }
$$

A straight forward calculation shows that

$$
\phi_{m, n}=\left\{\begin{array}{lc}
\frac{1}{m(m+1)} \sum_{j=1}^{m} j\left(x_{\sigma^{j}(n)}-x_{\sigma^{j-1}(n)}\right) & (m \geq 1) \\
x_{n} & (m=0) .
\end{array}\right.
$$


Note that for any sequence $x, y$ and scalar $\lambda$ we have

$$
\phi_{m, n}(x+y)=\phi_{m, n}(x)+\phi_{m, n}(y) \text { and } \phi_{m, n}(\lambda x)=\lambda \phi_{m, n}(x) .
$$

\section{Main Results}

Let $M$ be an Orlicz function, $p=\left(p_{m}\right)$ be any sequence of strictly positive real numbers, $r \geq 0$ and $(\mathrm{X}, \mathrm{q})$ be a seminorm space over the field $\mathbf{C}$ of complex number with seminorm $q$. Now we define the following sequence spaces,

$$
\begin{gathered}
B V_{\sigma}(M, p, q, r)=\left\{x=\left(x_{k}\right): \sum_{m=1}^{\infty} \frac{1}{m^{r}}\left[M\left(q\left(\frac{\left|\phi_{m, n}(x)\right|}{\rho}\right)\right)\right]^{p_{m}}\right. \\
<\infty, \text { uniformly in } n \text { and for som } \rho>0\} .
\end{gathered}
$$

For $M(x)=x$, we get

$$
\begin{gathered}
B V_{\sigma}(p, q, r)=\left\{x=\left(x_{k}\right): \sum_{m=1}^{\infty} \frac{1}{m^{r}}\left[\left(q\left(\left|\phi_{m, n}(x)\right|\right)\right)\right]^{p_{m}}\right. \\
<\infty, \text { uniformly in } n \text { and for som } \rho>0\} .
\end{gathered}
$$

For $p_{m}=1$ for all $m$, we get

$$
\begin{gathered}
B V_{\sigma}(M, q, r)=\left\{x=\left(x_{k}\right): \sum_{m=1}^{\infty} \frac{1}{m^{r}}\left[M\left(q\left(\frac{\left|\phi_{m, n}(x)\right|}{\rho}\right)\right)\right]\right. \\
<\infty, \text { uniformly in } n \text { and for som } \rho>0\} .
\end{gathered}
$$


For $r=0$, we get

$$
\begin{gathered}
B V_{\sigma}(M, p, q)=\left\{x=\left(x_{k}\right): \sum_{m=1}^{\infty}\left[M\left(q\left(\frac{\left|\phi_{m, n}(x)\right|}{\rho}\right)\right)\right]^{p_{m}}\right. \\
<\infty, \text { uniformly in } n \text { and for som } \rho>0\} .
\end{gathered}
$$

For $M(x)=x$ and $r=0$ we get

$$
\begin{gathered}
B V_{\sigma}(p, q)=\left\{x=\left(x_{k}\right): \sum_{m=1}^{\infty}\left[\left(q\left(\left|\phi_{m, n}(x)\right|\right)\right)\right]^{p_{m}}\right. \\
<\infty, \text { uniformly in } n \text { and for som } \rho>0\} .
\end{gathered}
$$

For $p_{m}=1$ for all $m$ and $r=0$ we get

$$
\begin{gathered}
B V_{\sigma}(M, q)=\left\{x=\left(x_{k}\right): \sum_{m=1}^{\infty}\left[M\left(q\left(\frac{\left|\phi_{m, n}(x)\right|}{\rho}\right)\right)\right]\right. \\
<\infty, \text { uniformly in } n \text { and for som } \rho>0\} .
\end{gathered}
$$

For $M(x)=x, p_{m}=1$ for all $m, r=0$ and $q(x)=|x|$ we get

$$
B V_{\sigma}=\left\{x=\left(x_{k}\right): \sum_{m=1}^{\infty}\left|\phi_{m, n}(x)\right|\right.
$$

$<\infty$, uniformly in $n$ and for som $\rho>0\}$. 
Theorem 2.1. The sequence space $B V_{\sigma}(M, p, q, r)$ is a linear space over the field $\mathbf{C}$ of complex numbers.

Proof. Let $x, y \in B V_{\sigma}(M, p, q, r)$ and $\alpha, \beta \in \mathbf{C}$. Then there exist positive numbers

$\rho_{1}$ and $\rho_{2}$ such that

$$
\sum_{m=1}^{\infty} \frac{1}{m^{r}}\left[M\left(q\left(\frac{\left|\phi_{m, n}(x)\right|}{\rho_{1}}\right)\right)\right]^{p_{m}}<\infty
$$

and

$$
\sum_{m=1}^{\infty} \frac{1}{m^{r}}\left[M\left(q\left(\frac{\left|\phi_{m, n}(y)\right|}{\rho_{2}}\right)\right)\right]^{p_{m}}<\infty \text { uniformly in } n
$$

Define $\rho_{3}=\max \left(2|\alpha| \rho_{1}, 2|\beta| \rho_{2}\right)$. Since $M$ is non-decreasing and convex we have

$$
\begin{aligned}
& \sum_{m=1}^{\infty} \frac{1}{m^{r}}\left[M\left(q\left(\frac{\left|\alpha \phi_{m, n}(x)+\beta \phi_{m, n}(y)\right|}{\rho_{3}}\right)\right)\right]^{p_{m}} \\
\leq & \sum_{m=1}^{\infty} \frac{1}{m^{r}}\left[M\left(q\left(\frac{\left|\alpha \phi_{m, n}(x)\right|}{\rho_{3}}+\frac{\left|\beta \phi_{m, n}(y)\right|}{\rho_{3}}\right)\right)\right]^{p_{m}}
\end{aligned}
$$

$\leq \sum_{m=1}^{\infty} \frac{1}{m^{r}} \frac{1}{2}\left[M\left(q\left(\frac{\left|\phi_{m, n}(x)\right|}{\rho_{3}}\right)\right)+M\left(q\left(\frac{\left|\beta \phi_{m, n}(y)\right|}{\rho_{3}}\right)\right)\right]<\infty$ uniformly in $n$.

This proves that $B V_{\sigma}(M, p, q, r)$ is a linear space over the field $\mathbf{C}$ of complex numbers. 
Theorem 2.2. For any Orlicz function $M$ and a bounded sequence $p=\left(p_{m}\right)$ of strictly positive real numbers, $B V_{\sigma}(M, p, q, r)$ is a paramormed (need not be total paranormed) space with

$$
\begin{gathered}
g(x)=\inf _{n \geq 1}\left\{\rho^{p_{n} / k}:\left(\sum_{m=1}^{\infty} m^{-r}\left[M\left(q\left(\frac{\left|\phi_{m, n}(x)\right|}{\rho}\right)\right)\right]^{p_{m}}\right)^{1 / K} \leq 1,\right. \\
\text { uniformly in } n\} .
\end{gathered}
$$

Where $K=\max \left(1, \sup p_{m}\right)$

Proof. It is clear that $g(x)=g(-x)$. Since $M(0)=0$, we get

$$
\inf \left\{\rho^{p_{n} / K}\right\}=0 \quad \text { for } x=0
$$

By using Theorem 1 , for $\alpha=\beta=1$, we get

$$
g(x+y) \leq g(x)+g(y)
$$

For the continuity of scalar multiplication let $l \neq 0$ be any complex number. Then by definition we have

$$
\begin{gathered}
g(l x)=\inf _{n \geq 1}\left\{\rho^{p_{n} / k}:\left(\sum_{m=1}^{\infty} m^{-r}\left[M\left(q\left(\frac{\left|\phi_{m, n}(l x)\right|}{\rho}\right)\right)\right]^{p_{m}}\right)^{1 / K} \leq 1,\right. \\
\text { uniformly in } n\} \\
g(l x)=\inf _{n \geq 1}\left\{(s|l|)^{p_{n} / k}:\left(\sum_{m=1}^{\infty} m^{-r}\left[M\left(q\left(\frac{\left|\phi_{m, n}(l x)\right|}{(s|l|)}\right)\right)\right]^{p_{m}}\right)^{1 / K} \leq 1,\right.
\end{gathered}
$$




$$
\text { uniformly in } n\}
$$

Where $s=\frac{\rho}{|l|}$. Since $|l|^{p_{n}} \leq \max \left(1,|l|^{H}\right)$, we have

$$
\begin{gathered}
g(l x) \leq \max \left(1,|l|^{H}\right) \inf _{n \geq 1}\left\{s^{p_{n} / k}:\left(\sum_{m=1}^{\infty} m^{-r}\left[M\left(q\left(\frac{\left|\phi_{m, n}(x)\right|}{s}\right)\right)\right]^{p_{m}}\right)^{1 / K} \leq 1,\right. \\
\text { uniformly in } n\} \\
=\max \left(1,|l|^{H}\right) g(x)
\end{gathered}
$$

and therefore $g(l x)$ converges to zero when $g(x)$ converges to zero in $B V_{\sigma}(M, p, q, r)$.

Now let $x$ be fixed element in $B V_{\sigma}(M, p, q, r)$. Then there exists $\rho>0$ such that

$$
\begin{gathered}
g(x)=\inf _{n \geq 1}\left\{\rho^{p_{n} / k}:\left(\sum_{m=1}^{\infty} m^{-r}\left[M\left(q\left(\frac{\left|\phi_{m, n}(x)\right|}{\rho}\right)\right)\right]^{p_{m}}\right)^{1 / K} \leq 1,\right. \\
\text { uniformly in } n\}
\end{gathered}
$$

Now

$$
\begin{gathered}
g(l x)=\inf _{n \geq 1}\left\{\rho^{p_{n} / k}:\left(\sum_{m=1}^{\infty} m^{-r}\left[M\left(q\left(\frac{\left|\phi_{m, n}(l x)\right|}{\rho}\right)\right)\right]^{p_{m}}\right)^{1 / K} \leq 1,\right. \\
\text { uniformly in } n\} \rightarrow 0
\end{gathered}
$$

as $l \rightarrow 0$. 
This completes the proof.

Theorem 2.3. Suppose that $0<p_{m} \leq t_{m}<\infty$ for each $m \in \mathbf{N}$ and $r \geq 0$. Then

(a) $B V_{\sigma}(M, p, q) \subseteq B V_{\sigma}(M, t, q)$,

(b) $B V_{\sigma}(M, q) \subseteq B V_{\sigma}(M, q, r)$.

Proof. $(i)$ suppose that $x \in B V_{\sigma}(M, p, q)$. This implies that

$$
\left[M\left(q\left(\frac{\left|\phi_{i, n}(x)\right|}{\rho}\right)\right)\right]^{p_{m}} \leq 1 \text { for sufficiently large values of } i \text {, say that }
$$

$i \geq m_{0}$ for some fixed $m_{0} \in \mathbf{N}$. Since $M$ is non- decreasing, we have

$$
\sum_{m=m_{0}}^{\infty}\left[M\left(q\left(\frac{\left|\phi_{i, n}(x)\right|}{\rho}\right)\right)\right]^{t_{m}} \leq \sum_{m=m_{0}}^{\infty}\left[M\left(q\left(\frac{\left|\phi_{i, n}(x)\right|}{\rho}\right)\right)\right]^{p_{m}}<\infty .
$$

Hence $x \in B V_{\sigma}(M, t, q)$.

The proof $(i i)$ is trivial.

The following result is consequence of the above result.

Corollary 1. If $0<p_{m} \leq 1$ for each $m$, then $B V_{\sigma}(M, p, q) \subseteq B V_{\sigma}(M, q)$. If $p_{m} \geq 1$ for all $m$, then $B V_{\sigma}(M, q) \subseteq B V_{\sigma}(M, p, q)$.

Theorem 2.4. The sequence space $B V_{\sigma}(M, p, q, r)$ is solid.

Proof. Let $x \in B V_{\sigma}(M, p, q, r)$. This implies that

$$
\sum_{m=1}^{\infty} m^{-r}\left[M\left(q\left(\frac{\left|\phi_{k, n}(x)\right|}{\rho}\right)\right)\right]^{p_{m}}<\infty
$$


Let $\left(\alpha_{m}\right)$ be sequence of scalars such that $\left|\alpha_{m}\right| \leq 1$ for all $m \in \mathbf{N}$. Then the result follows from the following inequality.

$$
\sum_{m=1}^{\infty} m^{-r}\left[M\left(q\left(\frac{\left|\alpha_{m} \phi_{k, n}(x)\right|}{\rho}\right)\right)\right]^{p_{m}} \leq \sum_{m=1}^{\infty} m^{-r}\left[M\left(q\left(\frac{\left|\phi_{k, n}(x)\right|}{\rho}\right)\right)\right]^{p_{m}}<\infty
$$

Hence $\alpha x \in B V_{\sigma}(M, p, q, r)$ for all sequences of scalar $\left(\alpha_{m}\right)$ with $\left|\alpha_{m}\right| \leq$ 1 for all $m \in \mathbf{N}$, whenever $x \in B V_{\sigma}(M, p, q, r)$.

From Theorem 4 and Lemma (1.1) we have:

Corollary 2. The sequence spaces $B V_{\sigma}(M, p, q, r)$ is monotone.

Theorem 2.5. Let $M_{1}$ and $M_{2}$ be Orlicz functions satisfying $\Delta_{2}$ condition and $r, r_{1}, r_{2} \geq 0$. Then we have

(i) If $r>1$ then $B V_{\sigma}\left(M_{1}, p, q, r\right) \subseteq B V_{\sigma}\left(M o M_{1}, p, q, r\right)$,

(ii) $B V_{\sigma}\left(M_{1}, p, q, r\right) \cap B V_{\sigma}\left(M_{2}, p, q, r\right) \subseteq B V_{\sigma}\left(M_{1}+M_{2}, p, q, r\right)$,

(iii) If $r_{1} \leq r_{2}$ then $B V_{\sigma}\left(M, p, q, r_{1}\right) \subseteq B V_{\sigma}\left(M, p, q, r_{2}\right)$.

Proof. (i). Since $M$ is continuous at 0 from right, for $\epsilon>0$ there exist $0<\delta<1$ such that $0 \leq c \leq \delta$ implies $M(c)<\epsilon$. If we define

$$
\begin{aligned}
& I_{1}=\left\{m \in \mathbf{N}: M_{1}\left(q\left(\frac{\left|\phi_{m, n}(x)\right|}{\rho}\right)\right) \leq \delta \text { for som } \rho>0\right\}, \\
& I_{2}=\left\{m \in \mathbf{N}: M_{1}\left(q\left(\frac{\left|\phi_{m, n}(x)\right|}{\rho}\right)\right)>\delta \text { for som } \rho>0\right\} .
\end{aligned}
$$


then, when $M_{1}\left(q\left(\frac{\left|\phi_{m, n}(x)\right|}{\rho}\right)\right)>\delta$, we get

$$
M\left[M_{1}\left(q\left(\frac{\left|\phi_{m, n}(x)\right|}{\rho}\right)\right)\right] \leq\left(\frac{2 M(1)}{\delta}\right)\left[M_{1}\left(q\left(\frac{\left|\phi_{m, n}(x)\right|}{\rho}\right)\right)\right] .
$$

Hence for $x \in B V_{\sigma}\left(M_{1}, p, q, r\right)$ and $r>1$

$$
\begin{gathered}
\sum_{m=1}^{\infty} m^{-r}\left[M o M_{1}\left(q\left(\frac{\left|\phi_{m, n}(x)\right|}{\rho}\right)\right)\right]^{p_{m}} \\
=\sum_{m \in I_{1}}^{\infty} m^{-r}\left[M o M_{1}\left(q\left(\frac{\left|\phi_{m, n}(x)\right|}{\rho}\right)\right)\right]^{p_{m}}+\sum_{m \in I_{2}}^{\infty} m^{-r}\left[M o M_{1}\left(q\left(\frac{\left|\phi_{m, n}(x)\right|}{\rho}\right)\right)\right]^{p_{m}} \\
\leq \sum_{m \in I_{1}}^{m \epsilon_{I_{2}}} m^{-r}[\epsilon]^{p_{m}}+\sum_{m \in I_{2}}^{\infty}\left(\frac{2 M(1)}{\delta}\right)\left[M_{1}\left(q\left(\frac{\left|\phi_{m, n}(x)\right|}{\rho}\right)\right)\right]^{p_{m}} \\
\leq \max \left(\epsilon^{h}, \epsilon^{H}\right) \sum_{m=1}^{\infty} m^{-r}+\max \left\{\left[\frac{2 M(1)}{\delta}\right]^{h},\left[\frac{2 M(1)}{\delta}\right]^{H}\right\}
\end{gathered}
$$

where $0<h=\inf p_{m} \leq p_{m} \leq H=\sup _{m} p_{m}<\infty$.

(ii) The proof follows from the following inequality:

$$
\begin{gathered}
m^{-r}\left[\left(M_{1}+M_{2}\right)\left(q\left(\frac{\left|\phi_{m, n}(x)\right|}{\rho}\right)\right)\right]^{p_{m}} \leq C m^{-r}\left[M_{1}\left(q\left(\frac{\left|\phi_{m, n}(x)\right|}{\rho}\right)\right)\right]^{p_{m}}+ \\
C m^{-r}\left[M_{2}\left(q\left(\frac{\left|\phi_{m, n}(x)\right|}{\rho}\right)\right)\right]^{p_{m}} \cdot
\end{gathered}
$$

(iii) The proof is straight forward.

Corollary 3. Let $M$ be an Orlicz function satisfying $\Delta_{2^{-}}$condition. Then we have

(i) If $r>1$ then $B V_{\sigma}(p, q, r) \subseteq B V_{\sigma}(M, p, q, r)$, 
(ii) $B V_{\sigma}(M, p, q) \subseteq B V_{\sigma}(M, p, q, r)$,

(iii) $B V_{\sigma}(p, q) \subseteq B V_{\sigma}(p, q, r)$,

(iv) $B V_{\sigma}(M, q) \subseteq B V_{\sigma}(M, q, r)$.

The proof is straight forward.

Acknowledgement: The author thanks the referee(s) for their valuable suggestions that improved the presentation of the paper.

\section{References}

[1] Z. U. Ahmad and M. Mursaleen, An application of banach limits, Proc. Amer. Math. Soc. 103, pp. 244-246, (1983).

[2] S. T. Chem, Geometry of Orlicz Spaces, Dissertationes Math. (The Institute of Mathematics, Polish Academy of Sciences) (1996).

[3] M. A. Krasnoselskii and Rutickii, Ya. B, Convex Functions and Orlicz Spaces, (Gooningen: P.Nordhoff Ltd.) (1961)(translation)

[4] J. Lindenstrauss and L. Tzafriri, On Orlicz sequence spaces, Israel J. Math., 10, pp. 379-390, (1971).

[5] G. G. Lorenz, A contribution to the theory of divergent sequences, Acta Math. 80, pp. 167-190, (1948).

[6] W. A. Luxemburg, Banach Function Spaces, Thesis (Delft), (1995).

[7] L. Maligranda, Orlicz Space and Interpolation, Seminar in Math.5 Campinas (1989).

[8] M. Mursaleen, Matrix Transformation Between some new sequence spaces, Houston J. Math., 9, pp. 505-509, (1983).

[9] M. Mursaleen, On some new invariant matrix methods of summability, Quart. J. Math., Oxford (2) 34, pp. 77-86, (1983).

[10] J. Musielak, Orlicz spaces and modular spaces, Lecture Notes in Math. 1034 (Springer-Verlag)(1983). 
[11] W. Orlicz, Über Raume $\left(L^{M}\right)$, Bulletin International del' Académie Polonaise de Sciences et des Letters, Série A, pp. 93-107, (1936).

[12] R. A. Raimi, Invariant means and invariant matrix method of summability, Duke Math. J., 30, pp. 81-94, (1963).

[13] M. M. Rao and Z.D.Ren, Theory of Orlicz spaces (New york, Basel, Hong Kong: Marcal Dekker Inc.) (1991)

[14] P. Schafer, Infinite matrices and invariant means Proc .Ammer. Math. Soc. 36, pp. 104-110, (1972).

[15] A. Wilansky, Summability through FunctionAlalysis,North-Holland Mathematical Studies, 85 (1984)

[16] K.Yosidak, Functional Analysis, Springer- Verlag, Berlian- Heidelberg Newyork., (1971)

\section{Aiyub}

Department of Mathematics, University of Bahrain, P. O. Box-32038, Kingdom of Bahrain e-mail : maiyub2002@gmail.com 